\title{
Differentiation of Human Embryonic Stem Cells into Engrafting Myogenic
} Precursor Cells.

\author{
Stalin Reddy Challa ${ }^{{ }^{*}}$ and Swathi Goli ${ }^{1}$ \\ ${ }^{1}$ Deportment of Pharmaceutics, Jangaon Institute of Pharmaceutical Sciences, India. \\ *Corresponding Author : Stalin Reddy Challa, Deportment of Pharmaceutics, Jangaon Institute of Pharmaceutical Sciences, India. \\ E-mail: challastalinreddy@gmail.com \\ Received date: March 16,2019;Accepted date : April 09,2019; Published date: April 11,2019. \\ Citation: : Stalin Reddy Challa and Swathi Goli, Differentiation of Human Embryonic Stem Cells into Engrafting Myogenic Precursor Cells J. Stem cell \\ Research and Therapeutics International, Doi: http://dx.doi.org/ 10.31579/ SCRTI/002.
}

Copyright : (c) 2019 Stalin Reddy Challa. This is an open-access article distributed under the terms of The Creative Commons Attribution License, which permits unrestricted use, distribution, and reproduction in any medium, provided the original author and source are credited.

\begin{abstract}
Degenerative muscle diseases affect muscle tissue integrity and function. Human embryonic stem cells (hESC) are an attractive source of cells to use in regenerative therapies due to their unlimited capacity to divide and ability to specialize into a wide variety of cell types. A practical way to derive therapeutic myogenic stem cells from hESC is lacking. In this study, we demonstrate the development of two serum-free conditions to direct the differentiation of hESC towards a myogenic precursor state. Using TGF 3 and PI3Kinase inhibitors in combination with bFGF we showed that one week of differentiation is sufficient for hESC to specialize into PAX3+/PAX7+ myogenic precursor cells. These cells also possess the capacity to further differentiate in vitro into more specialized myogenic cells that express MYOD, Myogenin, Desmin and MYHC, and showed engraftment in vivo upon transplantation in immunodeficient mice. Ex vivo myomechanical studies of dystrophic mouse hindlimb muscle showed functional improvement one month post-transplantation. In summary, this study describes a promising system to derive engrafting muscle precursor cells solely using chemical substances in serum-free conditions and without genetic manipulation.
\end{abstract}

Keywords: muscle tissue; human embryonic stem cells; myogenic precursor; immunodeficient

\section{Introduction}

Muscular dystrophies are debilitating degenerative muscle diseases caused by defective muscle proteins that disrupt normal homeostasis [1,2]. During embryogenesis Pax3 and Pax7-expressing cells drive muscle formation, growth and repair [3-8]. In the postnatal period, these cells are termed satellite cells -stem cells that remain quiescent beneath the basal lamina of muscle fibers under normal conditions. Tissue damage triggers these cells to proliferate and differentiate into myoblasts, leading to the formation of multinucleated myotubes and fibers [9-12]. However, dystrophic tissue induces repeated activation of satellite cells, ultimately leading to stem cell exhaustion, limiting the ability for further restorative myogenesis. Given their role in muscle formation, growth and repair, satellite cells, or other myogenic precursors with similar characteristics, would be attractive candidates for cell-based therapies for muscular dystrophies. Successful cell therapy would require myogenic precursor cells to: i. repopulate damaged muscle, ii. contribute to the formation of muscle fibers, and iii. lead to expression of normal muscle proteins in host tissue [13]. In addition, an important therapeutic benefit would be repopulation of the muscle stem cell niche to allow for ongoing homeostasis.

Embryonic stem cells (ESC) have the capacity to expand indefinitely, while maintaining the ability to differentiate into a wide variety of cell types. As such ESC may be useful for cell transplantation therapy or tissue engineering. However, a non-genetics method to obtain hESC derivatives with muscle regenerative abilities is lacking. Promising attempts to derive human myogenic precursors from pluripotent stem cells have relied on viral-mediated MYOD-expression [14-18], serum-containing media [14,15], use of demethylating agents [15], or selective multi-step cell culture approaches and cell sorting procedures that may be difficult to implement on a large-scale [16]. The most efficient methods described to date used genetic manipulation of human ESC (hESC) to overexpress the myogenic transcription factors, PAX3 and PAX7 [19]. Importantly, the use of genetically manipulated or virally induced cells, as well as cells exposed to serum that contains non-human components, may be difficult to use in human trials due to safety concerns.
Therefore, in order to establish alternative ways of driving early myogenic specification, we leveraged previous studies describing chemical compounds that stimulate and facilitate hESC differentiation toward paraxial mesoderm, the lineage responsible for limb and limb-associated skeletal muscle development. hESC can be chemically stimulated by activation of the bone morphogenetic protein (BMP) pathway, which is a key inducer of mesoderm formation during embryogenesis [14,20-24]. Treatment with the phosphatidylinositol 3-kinase (PI3K) inhibitor, Ly294002 hydrochloride (Ly), facilitates the differentiation of hESC by reducing self-renewal ability $[25,26]$. Additionally, paraxial mesoderm, the lineage responsible for limb muscle development, can be formed by brief stimulation with BMP and inhibition of the TGFb/Activin pathway using the small molecule, SB431542 (SB) [27]. This compound specifically inhibits the Activin pathway without affecting BMP signaling. It has been shown that inhibition of the TGFb/Activin pathway using SB up-regulates myogenic genes during hESC differentiation in defined culture conditions [14]. Another important factor in mesoderm formation is fibroblast growth factor (FGF), which also determines myogenic fate during development and plays a role in satellite cell activation [28-31]. Therefore, it is plausible that these components could be used to drive myogenesis in hESC cultures.

Based on these observations, we hypothesized that engrafting myogenic precursors could be derived from hESC by the sequential and coordinated manipulation of the BMP, TGF $\beta$ and FGF pathways. Importantly, we sought to prove that myogenic specification of hESC could be achieved without the use of allogeneic components in serum or genomic alteration. To this end, we tested whether myogenesis could be induced through embryoid body (EB) differentiation in medium containing basic fibroblast growth factor (bFGF), Ly and low levels of FGF, in combination with short exposure to BMP4 (referred to as $\mathrm{KFLy}(\mathrm{B}) \mathrm{S}$ medium). Separately, a combination of FGF and SB alone was tested for the ability to drive myogenesis (referred to as KFS medium). We determined the capacity of hESC-derived myogenic precursors to engraft in skeletal muscle upon transplantation in hindlimb muscles of SCID mice. In addition, we determined functional changes in transplanted muscle of mdx mice using ex vivo myomechanical analysis. 
The novel differentiation approach described in this study circumvents many of the limitations encountered in the transition of pluripotent stem cells into myogenic precursor cells, while enhancing our understanding of the mechanisms that govern stem cell differentiation and skeletal myoge.

\section{Materials and Methods}

\section{Human ESC culture and myogenic differentiation}

All work with hESC was done with the approval of UCSF Stem Cell Research Oversight Committee. The H9 hESC (WA09; WiCell) were maintained on irradiated CF1 mouse embryonic fibroblasts (MEF; Millipore, Temecula, CA) in KSR medium [Knockout Dulbecco's Modified Eagle Medium containing 20\% Knockout Serum Replacement, 2mM glutamine, $0.1 \mathrm{mM}$ nonessential amino acids, 0.1 $\mathrm{mM} \beta$-mercaptoethanol (all from Invitrogen, Carlsbad, CA)] supplemented with $12.5 \mathrm{ng} / \mathrm{ml}$ recombinant human fibroblast growth factor basic (bFGF; R\&D Systems, Minneapolis, MN) as previously described [32].

hESC colonies were detached after brief exposure to Collagenase IV ( $1 \mathrm{mg} / \mathrm{ml}$; Sigma-Aldrich, St. Lois, MO), washed and collected by settlement. Differentiation was initiated by embryoid body (EB) formation in pre-differentiation medium $\mathrm{KFLy}(\mathrm{B}) \mathrm{S}$ : KSR medium freshly supplemented with $20 \mathrm{ng} / \mathrm{ml}$ bFGF, $10 \mu \mathrm{M}$ Ly294,002 hydrochloride (Ly; Sigma-Aldrich), $10 \mu \mathrm{M}$ SB431542 hydrate (SB; Sigma-Aldrich), and for the first 36 hours supplementing with recombinant human bone morphogenetic protein-4 (BMP-4) (10 ng/ml; HumanZyme, Chicago, IL), or pre-differentiation medium KFS: KSR medium freshly supplemented with $40 \mathrm{ng} / \mathrm{ml}$ bFGF and 10 $\mu \mathrm{M}$ SB. Both pre-differentiation media were supplemented one time with $10 \mu \mathrm{M}$ Rho-associated protein kinase inhibitor (ROCKi) at the day of EB formation. SB and Ly were reconstituted in dimethylsulfoxide (DMSO) at $20 \mathrm{mM}$, and diluted to the final concentration in pre-differentiation medium. Human EBs were kept in suspension in ultra-low cluster 6-well plates (Costar, Corning, NY) and placed onto an orbital shaker (Daigger, Vernon Hills, IL) at a speed of $90 \mathrm{rpm}$. After 36 hours, medium was refreshed, which was repeated every other day until day six.

To assess the myocyte-forming potential of EB-derived myogenic precursor cells derived in KFLy(B)S and KFS (differentiation day 6) media, EBs were plated onto $12.5 \mu \mathrm{g} / \mathrm{cm} 2$ fibronectin (from human plasma; Sigma-Aldrich) in $0.1 \%$ gelatin-coated culture surfaces in predifferentiation medium to stimulate cell outgrowth. After two days, medium was replaced with muscle differentiation medium [DMEMhigh glucose, $10 \%$ fetal bovine serum (FBS; Hyclone, Thermo Scientific, South Logan, UT), 5\% horse serum (HS; also from Hyclone) supplemented with $5 \mathrm{ng} / \mathrm{ml} \mathrm{bFGF]} \mathrm{and} \mathrm{cultured} \mathrm{for} \mathrm{an}$ additional 4 days.

\section{Quantitative real-time PCR}

RNA was extracted from cells using the RNeasy Mini Kit including DNase treatment (Qiagen, Valencia, CA) according to the manufacturer's instruction. RNA concentration was measured using a Nanodrop ND-1000 Spectrophotometer (Nanodrop Technologies) to convert equal quantities of mRNA into cDNA using the Superscript ${ }^{\mathrm{TM}}$ III First-Strand Synthesis System (Invitrogen). Relative gene expression was determined using TaqMan Assay (Applied Biosytems) on an ABI 7300 Real-Time PCR system with the following human primer pairs (ABI): OCT4 (Hs00742896_s1); Brachyury (T) (HS00610080_m1); PAX3 (Hs00240950_m1); PAX7 (Hs00242962m1); MYOD (Hs00159528_m1); Desmin (Hs01090875_m1); Myogenin (Hs002311167_m1); MYHC (Hs00430042_m1) and GAPDH (4326317E). Cycle threshold (Ct) value for detection of gene of interest was normalized against $\mathrm{Ct}$ value of the housekeeping gene, human GAPDH, and relative changes were calculated according the $\Delta \Delta \mathrm{Ct}$ method. Results of relative fold expression were analyzed using GraphPad prism software in a twoway ANOVA with Bonferroni post-test.

\section{Immunostaining}

Immunofluorescence staining was performed on EB-outgrowths cultured in of KFLy(B)S or KFS with mouse anti-human Pax7 (undiluted), Myogenin (F5D; undiluted) and MyHC (MF20; 1:20) antibodies (all three supernatants from Developmental Studies Hybridoma Bank); mouse antihuman MyoD (5.8A; $20 \mu \mathrm{g} / \mathrm{ml}$; R\&D systems); or mouse anti-human Desmin (D33; $10 \mu \mathrm{g} / \mathrm{ml}$; Dako, Glostrup, Denmark). Cells were fixed in 4\% paraformaldehyde (PFA; EMS, Fort Washington, PA) for $15 \mathrm{~min}$. and permeabilized in $0.4 \%$ Triton-X100 for $10 \mathrm{~min}$. After blocking for $30 \mathrm{~min}$ in blocking solution [2\% normal goat serum (NGS; Dako), $1 \%$ bovine serum albumin (BSA; Sigma-Aldrich), $0.1 \%$ cold fish gelatin (SigmaAldrich), $0.1 \%$ Triton-X100, 0.05\% Tween-20 in phosphate buffered saline (PBS)], cells were subsequently incubated with the primary antibodies diluted in blocking solution for $1 \mathrm{hr}$. After washing (three times; 5 min in PBS with $0.05 \%$ Tween-20), primary antibodies were detected using Alexa Fluor ${ }^{\circledR}$ 488-labeled goat anti-mouse (1:200; Life Technologies, Grand Island, NY) for $30 \mathrm{~min}$. All steps were performed at room temperature (RT). Fluorescence microscopy was performed using a Nikon Eclipse TE300 microscope and Zeiss AxioScope Imager Z.2 with Apotome (Zeiss, Inc, Thornwood, NY).

\section{Intramuscular cell transplantation}

All experiments involving animals were done with the approval of the UCSF Institutional Animal Care and Use Committee. Hindlimbs of CB17.B6-PrkdcscidLyst bg/Crl (8-12 wk-old) and C57BL/10ScSnDmdmdx/J (X-linked muscular dystrophy; aged 6-8 weeks) mice (Jackson Laboratories, Bar Harbor, ME) were exposed to gamma radiation (5 Gy and $18 \mathrm{~Gy}$, respectively) three days before cell transplantation to inhibit muscle regeneration by host muscle cells. Mice cages were supplied with a water bottle containing an antibacterial oral suspension (200 mg sulfamethoxazole-40 mg trimethoprim/ $200 \mathrm{ml}$ water) from the day of irradiation through euthanasia. For intramuscular injection, EB-derived in KFLy(B)S or KFS were trypsinized and filtered through $40 \mu$ m nylon cell strainer (Gibco) to obtain a single cell suspension. One million cells cultured in $\operatorname{KFLy}(\mathrm{B}) \mathrm{S}$ or KFS were suspended in $50 \mu \mathrm{l}$ PBS (supplemented with $10 \mu \mathrm{M}$ ROCKi) and injected into the tibialis anterior (TA) muscle at two sites. As control, the contra-lateral muscle was injected with $50 \mu \mathrm{l}$ PBS with $10 \mu \mathrm{M}$ ROCKi. TA muscles were analyzed by immunohistochemistry one month post-transplantation, as described above.

\section{Immunohistochemistry}

TA muscles were embedded in OCT, snap-frozen in liquid nitrogen and stored at $-80^{\circ} \mathrm{C}$. Cryosections $(5 \mathrm{~mm})$ were fixed in acetone (ice cold, 10 min) and blocked in blocking solution [5\% NGS, 5\% Normal Donkey Serum (Dako), 5\% BSA, $0.1 \%$ cold fish gelatin, $0.1 \%$ Triton-X100, 0.05\% Tween-20 in PBS]. Sections were incubated with rabbit anti-human Dystrophin (1:2500; Novus Biologicals, Littleton, CO), chicken antimouse Laminin ( $2 \mathrm{mg} / \mathrm{ml}$; Abcam), mouse anti-human Desmin $(10 \mu \mathrm{g} / \mathrm{ml}$; D33; Dako), or mouse anti-human HLA-1 (W6/32; antigen determinant common to HLA-A, B and C; $4 \mathrm{mg} / \mathrm{ml}$; provided by Frances Brodsky, Dept. of Biopharmaceutical Sciences, UCSF) in blocking buffer for 1 hour. Alexa488/594-conjugated goat anti-mouse IgG, Alexa594-Donkey anti-Rabbit IgG, or Alexa488-Goat anti-Chicken IgG (1:250; all from Life Technologies) were used to detect primary antibodies. Sections were then washed and dehydrated in ethanol (70\%, 90\%, 100\%; 5 min each), airdried, and mounted in Vectashield with DAPI (Vector, Burlingame, CA). For immunohistochemical detection, sections were additionally blocked for endogenous peroxidase $(0.3 \% \mathrm{H} 2 \mathrm{O} 2$ in PBS, 20min). Primary antibody HLA-1 was detected by peroxidase labeled goat anti-mouse. Visualization was performed by a 3-amino-9-ethyl-carbazole (AEC; Sigma) substrate incubation for 4 minutes. Nuclei were counterstained with Mayer's haematoxyline (2 min, Fluka, Buchs, Zwitserland). All steps were performed at RT. Fluorescence microscopy was performed using a Nikon Eclipse TE300 microscope and Zeiss AxioScope Imager Z.2 with Apotome (Zeiss, Inc, Thornwood, NY). 


\section{Ex vivo myomechanical analysis of transplanted EDL muscle in $\mathrm{mdx}$ mouse model}

Ex vivo myography was performed to determine the increase in force production and to determine muscle fatigue level after cell transplantation into the extensor digitorum longus (EDL) muscle of mdx mouse. Myomechanical analysis was performed as previously described [33,34]. Force-frequency fatigue was measured by exposing muscle to a supramaximal stimulus train of 3-5 pulses $(300 \mathrm{msec}$ duration separated by $3 \mathrm{sec}$ ) at successive frequencies (30, 60, 100 and $140 \mathrm{~Hz}$ ), with 5 minute intervals between stimulations. Low-frequency time-fatigue was measured by supramaximal tetanic muscle stimulation at low frequency $(60 \mathrm{~Hz})$ for duration of $300 \mathrm{msec}$, repeated every 3 seconds for a period of 10 minutes. For myomechanical analysis, the left and right leg variables of an animal were averaged in each group and muscle mass, cross-sectional area, and length was measured. Significant differences were present between the means of each group compared to the PBS group. Multiple t-tests were performed with the number of comparisons adjusted by Bonferroni correction. A value of $\mathrm{p}<0.05$ was considered significant.

\section{Results}

\section{Inhibition of TGF $\beta$ pathway directs myogenic commitment}

To compare previously described, serum-containing methods for their capacity to induce the formation of myogenic precursors, we differentiated hESC by a selective culture approach using serumcontaining (10\% FBS) medium to enrich for CD73+ mesodermal stem cells followed by a second cell sort for expression of neural cell adhesion molecule (NCAM), a molecule also expressed by myogenic cells [16]. Furthermore, we differentiated hESC-EB in low serum conditions (2\% HS) [35-37] and high serum conditions (10\% FBS) during 2-4 weeks in culture [15]. We observed expression of PAX7 in cells derived through the selective multi-step methods (preCD73/NCAM sort) and when exposed to low and high serum conditions (Figure 1A). Addition of SB, a compound that inhibits the $\mathrm{TGFb}$ pathway, to cells differentiated in $10 \%$ FBS culture conditions resulted in markedly increased PAX7 levels (Figure 1A) ${ }^{*} p<0.01$. This indicated the myogenic stimulatory effect of TGFb inhibition in hESC. Next, we transiently stimulated the BMP pathway with BMP4 during EB differentiation combined with Ly and high levels of bFGF in serum-free conditions $(\operatorname{KFLy}(B))$. This resulted in high levels of PAX7 expression (Figure 1A) $p<0.001$. This was also seen when EB were exposed solely to high concentrations of FGF (KF) (Figure 1A) $p<0.001$.

$$
\text { Fig.1 }
$$

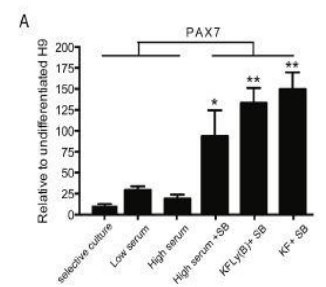

B

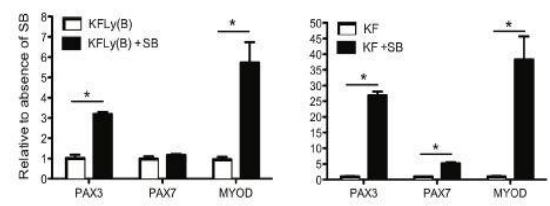

Figure 1: Differentiation of human embryonic stem cells along the myogenic lineage.

\section{Myogenic differentiation in EB culture}

We hypothesized that myogenic precursor cells would be more likely to engraft in host muscle, and have a greater chance of repopulating the stem cell niche, than fully differentiated myoblasts.
To monitor the stages of myogenic differentiation, we cultured hESC-EB in serum-free KFLy(B)S or KFS and examined myogenic gene expression by qRT-PCR at days 2, 4, 6 and 8, relative to undifferentiated hESC (day $0)$. OCT4 expression was used as an indicator of residual pluripotency after initiation of hESC differentiation. In both differentiation conditions, OCT4 expression fell dramatically by day 2 and further diminished through day 6 of differentiation (Figure 2) $p<0.001$.

$$
\text { Fig. } 2
$$

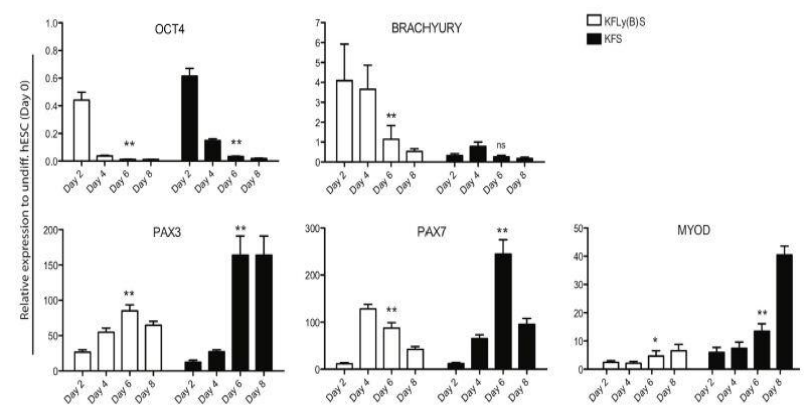

Figure 2: Myogenic commitment during 8 days of EB differentiation.

\section{KFLy(B)S- and KFS-derived precursors differentiate into myoblasts in vitro}

Directed differentiation of stem cells can be stimulated with exposure to specific extracellular matrix (ECM) components they would likely encounter in the stem cell niche [42]. This has specifically been shown for stem cell differentiation toward the myogenic lineage $[43,44]$. EB differentiated for 6 days in $\mathrm{KFLy}(\mathrm{B}) \mathrm{S}$ or KFS were plated in dishes coated with Matrigel, laminin or fibronectin to stimulate myogenic cell outgrowth. We observed that attachment to fibronectin-coated dishes, compared to Matrigel and laminin, more effectively promoted myogenic differentiation (data not shown). Therefore, fibronectin coating was used to evaluate the myogenic potential of EB-outgrowth cells (EB-O) during the subsequent 6 days in muscle differentiation medium.

\section{Discussion}

PAX3 and PAX7 are critical factors in embryonic skeletal myogenesis and participate after birth in muscle homeostasis. They are, therefore, important indicators of a myogenic precursor stage that harbors regenerative capacity. Several methods have been published describing the myogenic differentiation of hESC, however, limitations of published methods include the use of non-human serum and genetic manipulation that may hamper therapeutic application due to safety concerns $[48,49]$. This study underscores that myogenesis can be achieved in hESC cultures in a short time frame when exposed to appropriate developmental cues. We have demonstrated two approaches to derive PAX3- and PAX7expressing myogenic precursors from hESC using a novel sequence of chemical compounds that direct a myogenic cell fate. Upon transplantation, these muscle precursor cells engrafted into muscle, formed new fibers, and improved muscle strength.

In the course of this study a publication revealed the success of a chemically defined culture system in transgenic zebrafish that directs skeletal myogenesis of human induced pluripotent stem cells [50]. Therefore, the combination of pathway inhibitors and stimulators described in this study provide an effective platform to advances approaches to muscle differentiation from hESC. Among the benefits are the avoidance of the use of serum and genetic alteration to promote gene expression. Furthermore, we showed that hESC entered the myogenic program in less then one-week making this approach faster than other published methods applying hESC differentiation during 2-4 weeks. Additionally, lacking selective cell culture approaches contributed to a method that is costs effective and easy.

In mdx mice, transplantation with hESC-derived precursors derived using these methods resulted in improved mechanical force generation and decreased fatigue. 
Both muscles injected with $\operatorname{KFLy}(B) S$ and KFS-derived cells demonstrated higher maximal tension than muscle injected with PBS or EDL that was not injected with cells, indicating improved functional capacity. Interestingly, EDL from mice injected with KFLy(B)S-derived cells demonstrated greater maximal tetanic tension, compared to both EDL from mice injected with PBS and KFS-derived cells and EDL that was not injected. However, force-frequency analysis demonstrated decreased fatigue in muscle from mice injected with KFS-derived cells compared to the other groups. The mechanisms that might account for these differences are unclear, but it is intriguing that the ESC-derived precursors could alter functional capacity by affecting different muscle properties (e.g strength vs endurance). Most importantly, however, the findings of the myomechanical analysis indicate preliminary proof-of-concept for functional myogenic potential for these hESC-derived precursor cells.

\section{Conclusion}

We have shown that a short exposure of hESC to chemically defined media promotes differentiation of hESC into engrafting myogenic precursor cells that can contribute to the formation of new fibers in vivo and improve muscle strength as analyzed ex vivo. These techniques are less laborious than previously published methods, and the avoidance of xenobiotic agents and serum may mitigate safety concerns. Further studies to assess durability of transplanted cells and newly formed fibers, as well as their contribution to muscle homeostasis over time are warranted.

\section{References}

1. Emery AE. The muscular dystrophies. Lancet. 2002; 359(9307): 687-95.

2. Matsumura K, KP Campbell. Dystrophin-glycoprotein complex: its role in the molecular pathogenesis of muscular dystrophies. Muscle Nerve. 1994; 17(1): 2-15.

3. Kassar-Duchossoy L, Giacone E, Gayraud-Morel B, Jory A, Gomès D, Tajbakhsh S. et al., Pax3/Pax7 mark a novel population of primitive myogenic cells during development. Genes Dev. 2005; 19(12): 1426-31.

4. Zammit PS, Relaix F, Nagata Y, Ruiz AP, Collins CA, Partridge TA, et al., Pax7 and myogenic progression in skeletal muscle satellite cells. J Cell Sci. 2006; 119(Pt 9): 1824-32.

5. Relaix F, Rocancourt D, Mansouri A, Buckingham M. A Pax3/Pax7-dependent population of skeletal muscle progenitor cells. Nature. 2005; 435(7044): 948-53.

6. Seale P, Sabourin LA, Girgis-Gabardo A, Mansouri A, Gruss P, Rudnicki MAeeee. Cell. 2000; 102(6): 777-86.

7. Oustanina, S., G. Hause, T. Braun, Pax7 directs postnatal renewal and propagation of myogenic satellite cells but not their specification. Embo J. 2004; 23(16): 3430-9.

8. Collins CA, Olsen I, Zammit PS, Heslop L, Petrie A, Partridge TA, et al., Stem cell function, self-renewal, and behavioral heterogeneity of cells from the adult muscle satellite cell niche. Cell. 2005; 122(2): 289-301.

9. Mauro A. Satellite cell of skeletal muscle fibers. J Biophys Biochem Cytol. 1961; 9: 493-5.

10. Braun T, Rudnicki MA, Arnold HH, Jaenisch R. Targeted inactivation of the muscle regulatory gene Myf-5 results in abnormal rib development and perinatal death. Cell. 1992. 71(3): 369-82.

11. Rudnicki MA, Braun T, Hinuma S, Jaenisch R. Inactivation of MyoD in mice leads to up-regulation of the myogenic HLH gene Myf-5 and results in apparently normal muscle development. Cell. 1992. 71(3): 383-90.

12. Tajbakhsh S, Rocancourt D, Cossu G, Buckingham M. Redefining the genetic hierarchies controlling skeletal myogenesis: Pax-3 and Myf-5 act upstream of MyoD. Cell. 1997; 89(1): 127-38.

13. Wilschut KJ, VB Ling, HS Bernstein. Concise review: stem cell therapy for muscular dystrophies. Stem Cells Transl Med, 2012; 1(11): 833-42. doi: 10.5966/sctm.2012-0071.
14. Mahmood A, Harkness L, Schrøder HD, Abdallah BM, Kassem M. Enhanced differentiation of human embryonic stem cells to mesenchymal progenitors by inhibition of TGF-beta/activin/nodal signaling using SB-431542. J Bone Miner Res. 2010; 25(6): 1216-33.

15. Zheng JK, Wang Y, Karandikar A, Wang Q, Gai H, Liu AL, et al., Skeletal myogenesis by human embryonic stem cells. Cell Res. 2006; 16(8): 713-22.

16. Barberi T, Bradbury M, Dincer Z, Panagiotakos G, Socci ND, Studer L. Derivation of engraftable skeletal myoblasts from human embryonic stem cells. Nat Med. 2007; 13(5): 642-8.

17. Goudenege S, Lebel C, Huot NB, Dufour C, Fujii I, Gekas J, et al., Myoblasts derived from normal hESCs and dystrophic hiPSCs efficiently fuse with existing muscle fibers following transplantation. Mol Ther. 2012; 20(11): 2153-67.

18. Rao L, Tang W, Wei Y, Bao L, Chen J, Chen H, et al., Highly efficient derivation of skeletal myotubes from human embryonic stem cells. Stem Cell Rev. 2012; 8(4): 1109-19. doi: 10.1007/s12015-0129413-4.

19. Darabi R, Arpke RW, Irion S, Dimos JT, Grskovic M, Kyba M, et al., Human ES- and iPS-Derived Myogenic Progenitors Restore DYSTROPHIN and Improve Contractility upon Transplantation in Dystrophic Mice. Cell Stem Cell. 2012; 10(5): 610-9. doi: 10.1016/j.stem.2012.02.015

20. Zhang P, Li J, Tan Z, Wang C, Liu T, Chen L, et al. Short-term BMP-4 treatment initiates mesoderm induction in human embryonic stem cells. Blood. 2008; 111(4): 1933-41.

21. Murry, CE, G Keller. Differentiation of embryonic stem cells to clinically relevant populations: lessons from embryonic development. Cell. 2008; 132(4): 661-80. doi: 10.1016/j.cell.2008.02.008.

22. Winnier G, Blessing $M$, Labosky PA, Hogan BL. Bone morphogenetic protein-4 is required for mesoderm formation and patterning in the mouse. Genes Dev. 1995; 9(17): 2105-16.

23. Yu P, Pan G, Yu J, Thomson JA. FGF2 sustains NANOG and switches the outcome of BMP4-induced human embryonic stem cell differentiation. Cell Stem Cell. 2011; 8(3): 326-34. doi: 10.1016/j.stem.2011.01.001

24. Bernardo AS, Faial T, Gardner L, Niakan KK, Ortmann D, Senner $\mathrm{CE}$, et al. BRACHYURY and CDX2 mediate BMP-induced differentiation of human and mouse pluripotent stem cells into embryonic and extraembryonic lineages. Cell Stem Cell. 2011; 9(2): 144-55. doi: 10.1016/j.stem.2011.06.015.

25. McLean AB, D'Amour KA, Jones KL, Krishnamoorthy M, Kulik MJ, Reynolds DM, et al., Activin a efficiently specifies definitive endoderm from human embryonic stem cells only when phosphatidylinositol 3-kinase signaling is suppressed. Stem Cells. 2007; 25(1): 29-38.

26. Paling NR, Wheadon H, Bone HK, Welham MJ. Regulation of embryonic stem cell self-renewal by phosphoinositide 3-kinasedependent signaling. J Biol Chem, 2004. 279(46): 48063-70.

27. Cheung C, Bernardo AS, Trotter MW, Pedersen RA, Sinha S. Generation of human vascular smooth muscle subtypes provides insight into embryological origin-dependent disease susceptibility. Nat Biotechnol. 2012; 30(2): 165-73.

28. Lagha M, Kormish JD, Rocancourt D, Manceau M, Epstein JA, Zaret KS, et al., Pax3 regulation of FGF signaling affects the progression of embryonic progenitor cells into the myogenic program. Genes Dev. 2008; 22(13): 1828-37. doi: 10.1101/gad.477908

29. Chakkalakal JV, Jones KM, Basson MA, Brack AS. The aged niche disrupts muscle stem cell quiescence. Nature. 2012; 490(7420): 35560. doi: 10.1038/nature11438

30. Yang L, Soonpaa MH, Adler ED, Roepke TK, Kattman SJ, Kennedy $\mathrm{M}$, et al. Human cardiovascular progenitor cells develop from a $\mathrm{KDR}+$ embryonic-stem-cell-derived population. Nature. 2008; 453(7194): 524-8. doi: 10.1038/nature06894.

31. Yook JY, Kim MJ, Son MJ, Lee S, Nam Y, Han YM, et al., Combinatorial activin receptor-like kinase/Smad and basic fibroblast growth factor signals stimulate the differentiation of human embryonic stem cells into the cardiac lineage. Stem Cells Dev. 2011. 20(9): 1479-90. doi: 10.1089/scd.2010.0392. 
38. Rudnicki MA, Schnegelsberg PN, Stead RH, Braun T, Arnold HH, Jaenisch R, et al., MyoD or Myf-5 is required for the formation of skeletal muscle. Cell. 1993; 75(7): 1351-9.

39. Kassar-Duchossoy L, Gayraud-Morel B, Gomès D, Rocancourt D, Buckingham M, Shinin V, et al. Mrf4 determines skeletal muscle identity in Myf5:Myod double-mutant mice. Nature. 2004; 431(7007): 466-71.

40. Horsley V, Pavlath GK. Pavlath, Forming a multinucleated cell: molecules that regulate myoblast fusion. Cells Tissues Organs. 2004; 176(1-3): 67-78.

41. Dhawan J, Rando TA. Stem cells in postnatal myogenesis: molecular mechanisms of satellite cell quiescence, activation and replenishment. Trends Cell Biol. 2005; 15(12): 666-73.

42. Engler AJ, Sen S, Sweeney HL, Discher DE. Matrix elasticity directs stem cell lineage specification. Cell. 2006. 126(4): 677-89.

43. Engler AJ, Griffin MA, Sen S, Bönnemann CG, Sweeney HL, Discher DE. Myotubes differentiate optimally on substrates with tissue-like stiffness: pathological implications for soft or stiff microenvironments. J Cell Biol. 2004; 166(6): 877-87.

44. Wilschut KJ, Haagsman HP, Roelen BA. Extracellular matrix components direct porcine muscle stem cell behavior. Exp Cell Res. 2010; 316(3): 341-52. doi: 10.1016/j.yexcr.2009.10.014 